\title{
20 años de investigación sobre Gobierno Corporativo Internacional
}

\author{
Laura VIVAS-CRISOL \\ IQS School of Management-Universitat Ramon Llull \\ Departamento de Economía y Finanzas \\ lauravivasc@iqs.edu \\ Mónica MARTÍNEZ-BLASCO \\ IQS School of Management-Universitat Ramon Llull \\ Departamento de Economía y Finanzas \\ monica.martinez@iqs.edu \\ Josep GARCÍA-BLANDÓN \\ IQS School of Management-Universitat Ramon Llull \\ Departamento de Economía y Finanzas \\ josep.garcia@iqs.edu
}

Recibido: Noviembre 2011

Aceptado: Junio 2012

Resumen: El Gobierno Corporativo Internacional es un tema de creciente interés en el área de Economía y Finanzas, ya que a pesar de todos los resultados que han supuesto décadas de investigación, este tema sigue estando en el centro de atención y en ello mucho ha tenido que ver la crisis financiera desatada a finales del 2007. El presente artículo analiza la producción científica sobre gobierno corporativo internacional desde que se publicó el primer artículo en 1992 hasta 2010. Los datos se han obtenido de la base de datos ISI Web of Science dando por resultado 338 artículos que se han analizado obteniendo los artículos más citados, la evolución temporal del número de publicaciones, los autores y revistas más citados y el índice de colaboración científica.

Palabras clave: Gobierno Corporativo Internacional; producción científica; ISI WoS; índice de colaboración.

\section{0 years of International Corporate Governance research}

\begin{abstract}
International Corporate Governance is a topic of growing interest. Despite all the results that have led years of research, this issue remains an interesting topic in Economics and Finance. This article examines the scientific literature on international corporate governance since the first article was published in 1992. The data is obtained from the database ISI Web of Science, resulting in 338 articles that have been analyze to obtain the most cited articles, the evolution in the number of publications, the authors and journals cited and Index of Scientific Collaboration.
\end{abstract}

Keyword: International corporate governance; scientific production; ISI WoS, Scientific; Collaboration Index. 


\section{INTRODUCCIÓN}

A mediados de los años setenta Jensen y Meckling (1976) intentan dar respuesta a una pregunta que se plantea de forma persistente al analizar el gobierno de las empresas: cuando la propiedad y el control de una compañía no coinciden ¿cómo conseguir minimizar los conflictos de interés que se plantean entre los propietarios y los administradores? Esta pregunta, que a finales de los años setenta quedaba limitada a la relación entre los directores de las compañías y sus accionistas, se amplió sustancialmente durante la década de los noventa, con la incorporación de otros grupos de interés que también se podían ver afectados por dichos conflictos. Este hecho hace que se desarrolle una nueva línea de investigación en finanzas corporativas conocida como "Gobierno Corporativo". En la que es probablemente una de las definiciones más utilizada para explicar el concepto de gobierno corporativo, Shleifer y Vishny (1997) lo presentan como "todo aquello que permite a los suministradores de recursos financieros de las empresas asegurarse un rendimiento a su inversión".

Como en casi todos los temas de investigación que atañen a la economía financiera, la investigación sobre el gobierno de las empresas se inicia en Estados Unidos durante la década de los años 70 y se extiende durante la década de los 80 bajo la perspectiva de la teoría de la agencia. No será hasta los años 90 cuando se generalice la investigación al resto de países, dado que los numerosos escándalos financieros y contables que se registraron en múltiples países con sistemas financieros desarrollados que hicieron proliferar leyes, normativas $y$ recomendaciones para proteger a los accionistas y al resto de grupos de interés relacionados con la empresa de los posibles abusos de poder por parte de los directivos. Así, a finales de los noventa, el gobierno corporativo constituye uno de los temas más investigados en los ámbitos académicos de economía y empresa, (Correa et al., 2009) dando lugar a una investigación cada vez más especializada.

La línea de investigación que analiza las diferencias sobre gobierno corporativo en función del origen legal de los países se conoce como Gobierno Corporativo Internacional (GCI). Las publicaciones sobre GCI comenzaron en la década de los noventa coincidiendo con la apertura del debate en Estados Unidos sobre la eficiencia de los diferentes sistemas de gobierno corporativo existentes. Así, en sus inicios, los artículos sobre GCI analizaban individualmente diferentes países que no fuesen Estados Unidos poniendo en evidencia las diferencias existentes entre los mecanimos de gobierno corporativo empleados y su eficiencia en función del país analizado: lo que Denis y McConnell (2003) denominan la primera generación de investigación sobre GCI. Los mismos autores identifican una segunda etapa de la investigación donde los estudios se hacen más complejos, más amplios, y en los cuales se analizan varios países a la vez para determinar las diferencias subyacentes en sus sistemas de gobierno corporativo. 
Filatotchev y Boyd (2009) enumeran cuatro problemas por los cuales la investigación de gobierno corporativo desde una perspectiva internacional no se generalizó hasta mediados de los noventa. El primer problema fue el difícil acceso a la información contable-financiera fuera de los EE.UU, hecho que fue revertido a mediados de los noventa gracias al acceso a las bases de datos financieras y a la gran divulgación de los cambios en las normativas contables y financieras. El segundo problema fue la procedencia de los autores, los primeros artículos que investigan sobre GCI eran esencialmente estadounidenses y británicos. Este problema se superó con la incorporación de investigadores sobre gobierno corporativo que empezaron a investigar en sus respectivos países y comparándolos internacionalmente. Otra problemática a la cual hacia frente esta línea de investigación era las grandes diferencias entre países ya que tan solo se intentaba investigar qué modelo de gobierno corporativo era el más adecuado. Y por último, el aspecto interdisciplinario de este tema, ya que puede abordarse desde disciplinas como la legal, la económica, ética, sociológica, etc.

Pese a la extensa literatura científica existente sobre gobierno corporativo y más concretamente sobre gobierno corporativo internacional, existen pocos trabajos que analicen su producción científica. 20 años de producción científica nos devuelve una cantidad de trabajos que empieza a ser interesante analizar y que, hasta donde nosotros hemos llegado, aún no se ha hecho. Bien es cierto que existen trabajos que recopilan y resumen las ideas más destacadas de grandes autores sobre este tema, mostrando de esta forma el avance del conocimiento. Chew (1997) realiza una revisión de la literatura en la que analiza 28 artículos relacionados con el gobierno corporativo de Estados Unidos, Japón y Europa. Ese mismo año Shleifer y Vishny (1997) resumen las principales líneas y autores sobre gobierno corporativo dándole especial importancia a la protección jurídica de los inversores y a la concentración de la propiedad en los sistemas de gobierno corporativo en todo el mundo. Becht et al. (2003), revisan la investigación teórica y empírica sobre los mecanismos de control corporativo, examinan las principales instituciones legales y reguladoras en diferentes países y analizan la literatura comparada sobre gobierno corporativo. Tal y como se ha mencionado en párrafos anteriores, Dennis y McConnell (2003) repasan la literatura sobre GCI segmentándola en dos generaciones; la primera generación de artículos basados fundamentalmente en estudios donde se analiza de forma individual el gobierno corporativo en distintos países y una segunda generación de artículos donde se considera el impacto de los diferentes sistemas legales en la estructura y el funcionamiento del gobierno corporativo comparando estos sistemas entre países. Más recientemente, Bedchuck y Weisbach (2009) estudian el estado de la investigación sobre gobierno corporativo, dividiendo la investigación en siete áreas, discutiendo la importancia de cada una de ellas, destacando la contribución que los autores aportan a cada área y mencionando las futuras líneas de investigación. Durisin y Puzone (2009), realizan una revisión de la literatura sobre 
gobierno corporativo con el objetivo de delimitar si la investigación sobre gobierno corporativo constituye en sí misma una disciplina. Por último cabe destacar el trabajo de Richart et al (2011) donde se realiza un estudio bibliométrico de 2.147 publicaciones sobre gobierno corporativo sin entrar a analizar las diferentes líneas de investigación.

El presente trabajo continua la línea de investigación abierta por el artículo anteriormente mencionado de Richart et al. (2011). En él se establece que la investigación sobre gobierno corporativo se fundamenta principalmente en el artículo de La Porta et al (1998), siendo este el artículo el más citado, en el que justifican que un factor determinante en las medidas y leyes que regulan el gobierno corporativo es el contexto institucional de cada país, siendo más estrictos los países de tradición legal Común que los países de tradición legal Civil. Por esta razón, resulta interesante realizar un nuevo estudio bibliométrico sobre GCI como subdisciplina del Gobierno Corporativo, para establecer si la comunidad científica ha contrastado los resultados encontrados por La Porta et al. (1998).

El objetivo de este trabajo es realizar un recorrido por los últimos 20 años de producción científica sobre GCI con la finalidad de mostrar su evolución y aportar resultados descriptivos y bibliométricos que sean útiles para fundamentar futuras investigaciones. El análisis nos permitirá obtener los artículos más citados, los autores que están especializados en este tema y las revistas de referencia en el área y así poder hacer una composición de lugar de lo que ha sido la investigación científica en este ámbito. El presente trabajo se realiza con la ayuda de la base de datos científica de referencia ISI Web of Science y con datos actualizados hasta 2010.

El trabajo está estructurado de la siguiente forma: en el siguiente apartado se encuentra la metodología donde se explica la fuente de obtención de los datos y su tratamiento matemático, en el punto 3 se presentan los resultados obtenidos del análisis y en el punto 4 se exponen las principales conclusiones.

\section{METODOLOGÍA}

Los datos necesarios para poder cumplir con el objetivo planteado han sido obtenidos de la base de datos ISI Web of Science (ISI WoS) que recoge información bibliográfica de revistas indexadas en Science Citation Index Expanded, Sciences Citation Index y el Arts \& Humanities Citation Index, lo que supone cerca de 10.000 revistas.

La búsqueda se realizó introduciendo en la línea Topic de la base de datos ISI WoS el término International Corporate Governance sin limitaciones en la fecha de inicio y estableciendo como fecha final 2010. A pesar de que la búsqueda principal de artículos se realizó en el mes de noviembre de 2010, ésta se actualizó con las incorporaciones de publicaciones correspondientes a 2010 que fueron introducidas hasta finales de febrero de 2011. Esta búsqueda se refinó 
posteriormente excluyendo todo tipo de documentos que no fueran artículos dando lugar a una muestra final de 338 artículos.

Toda la información que suministra ISI WoS de cada artículo, incluido el resumen y las referencias citadas, se descargó en un archivo delimitado por tabulaciones desde la página web mediante la aplicación que hace posible exportar la información, incluyendo el registro completo y las referencias citadas. Una vez volcada toda esta información a una hoja de cálculo se procedió a la extracción y ordenación de los datos de forma que fuesen útiles para el estudio.

\section{RESULTADOS}

\subsection{ARTÍCULOS}

El número de artículos publicados sobre GCI sigue una tendencia creciente en los últimos años tal y como se puede apreciar en el gráfico 1. El primer artículo que aparece publicado data de 1992 (Demb y Neubaue, 1992) y trata sobre las diferencias y las similitudes de los consejos de administración de diferentes países.

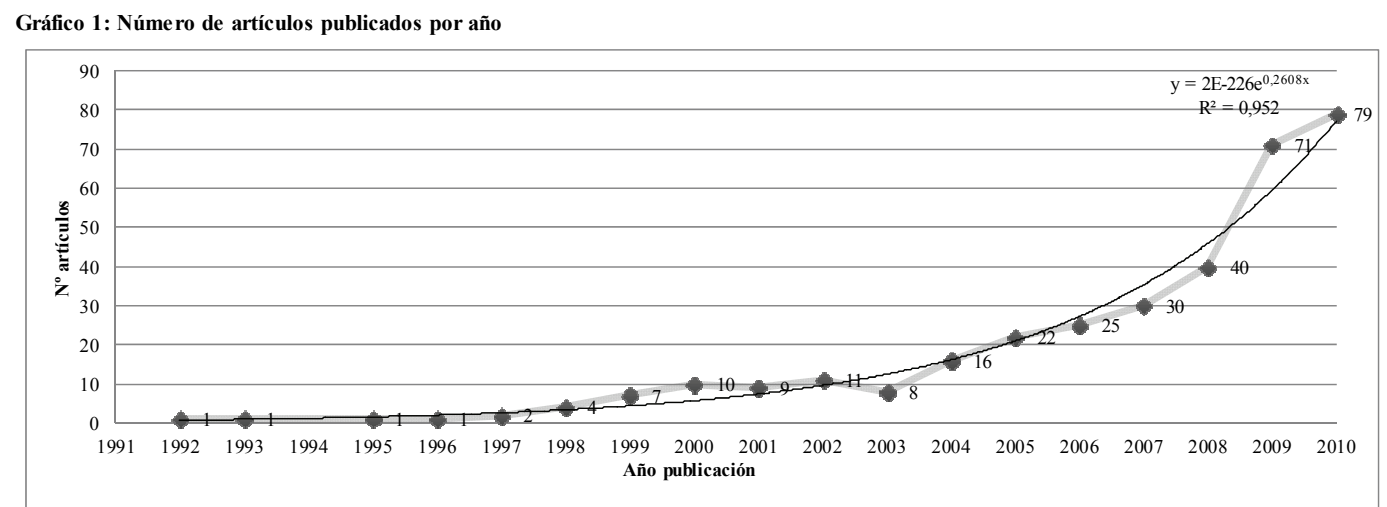

Fuente: Elaboración propia a partir de los datos de ISI WoS.

En el año 2004 se aprecia un gran aumento de las publicaciones, duplicándose durante ese año la cifra de artículos respecto al año anterior. El 83\% de la producción científica sobre GCI (280 artículos de 338) se ha publicado precisamente en esta etapa, siendo el 2010 el año con mayor número de publicaciones $(21,19 \%$ de la muestra). Aplicando la ley de crecimiento exponencial de Price sobre la producción anual de artículos, función que se muestra en la figura 1 , se obtiene una tasa de crecimiento anual del $29,70 \%$.

Por lo que respecta a los artículos más utilizados por el resto de autores para fundamentar sus investigaciones (Tabla 1), se constata que el artículo más citado es el correspondiente a Ball et al. (2000) con 241 citas acumuladas. En él, los 
autores argumentan que las diferencias contables internacionales afectan en la manera en que se incorporan los ingresos económicos y compara las diferencias en función de la adscripción de cada país a su sistema legal.

Tabla 1: Artículos más relevantes de Gobierno Corporativo Internacional según ISI WoS

\begin{tabular}{|l|l|l|l|l|l|l|}
\hline Autores & Institución & Año & Título & Revista & $\begin{array}{l}\text { Citas } \\
\text { (ISI } \\
\text { WoS) }\end{array}$ & $\begin{array}{c}\text { Media } \\
\text { citas } \\
\text { año }\end{array}$ \\
\hline $\begin{array}{l}\text { Ball } \\
\text { Kothari } \\
\text { Robin }\end{array}$ & $\begin{array}{l}\text { University of Chicago, EEUU } \\
\text { Sloan School of managment (MIT), EEUU } \\
\text { Rochester Institute of technology, EEUU }\end{array}$ & 2000 & $\begin{array}{l}\text { The effect of international } \\
\text { institutional factors on } \\
\text { properties of accounting }\end{array}$ & $\begin{array}{l}\text { Journal of } \\
\text { Accounting \& } \\
\text { Economics }\end{array}$ & 241 & 21,91 \\
\hline $\begin{array}{l}\text { Dyck } \\
\text { Zingales }\end{array}$ & $\begin{array}{l}\text { Harvard Business School, EEUU } \\
\text { University of Chicago, EEUU }\end{array}$ & 2004 & $\begin{array}{l}\text { Private benefits of control: An } \\
\text { international comparison }\end{array}$ & $\begin{array}{l}\text { Journal of } \\
\text { Finance }\end{array}$ & 210 & 30,00 \\
\hline $\begin{array}{l}\text { Doidge } \\
\text { Karolyi } \\
\text { Stulz }\end{array}$ & $\begin{array}{l}\text { Rotman School of Management, Canada } \\
\text { Fisher College of Business, EEUU } \\
\text { Fisher College of Business, EEUU }\end{array}$ & 2004 & $\begin{array}{l}\text { Why are foreign firms listed in } \\
\text { the US worth more? }\end{array}$ & $\begin{array}{l}\text { Journal of } \\
\text { Financial } \\
\text { Economics }\end{array}$ & 140 & 20,00 \\
\hline $\begin{array}{l}\text { Lins } \\
\text { Servaes }\end{array}$ & $\begin{array}{l}\text { University of Utah, EEUU } \\
\text { London Business School, R.U }\end{array}$ & 1999 & $\begin{array}{l}\text { International evidence on the } \\
\text { value of corporate } \\
\text { diversification }\end{array}$ & $\begin{array}{l}\text { Journal of } \\
\text { Finance }\end{array}$ & 80 \\
\hline $\begin{array}{l}\text { Klapper } \\
\text { Love }\end{array}$ & $\begin{array}{l}\text { The World Bank, EEUU } \\
\text { The World Bank, EEUU }\end{array}$ & $\begin{array}{l}\text { Corporate governance, } \\
\text { investor protection, and } \\
\text { performance in emerging }\end{array}$ & $\begin{array}{l}\text { Journal of } \\
\text { Corporate } \\
\text { Finance }\end{array}$ & 7,27 \\
\hline
\end{tabular}

Fuente: Elaboración propia a partir de los datos de ISI WoS.

El segundo artículo en número de citas corresponde a Dyck y Zingales (2004) con 210 citas y en él se establece la existencia de una correlación inversa entre los beneficios privados de control y un mercado poco desarrollado, propiedad concentrada y numerosas privatizaciones y al contrario, que en existencia de un alto cumplimiento de la ley, una alta difusión en prensa, gran competencia y gran cumplimiento tributario los beneficios de control son menores. A mucha más distancia en número de citas de estos dos primeros artículos se encuentra Doidge et al. (2004) con 140, quienes argumentan que los accionistas mayoritarios de empresas extranjeras que cotizan en los EE.UU tienen menores beneficios de control. Por lo tanto se debe tratar de compañías donde los intereses de los accionistas mayoritarios estén más alineados con los intereses del resto de accionistas. También demuestra que estas compañías que cotizan en su mercado de origen y EE.UU aprovechan mejor las oportunidades de crecimiento porque tan solo una mínima parte de los flujos de caja será apropiada por los accionistas mayoritarios. El cuarto artículo más citado es de Lins y Servaes (1999) con 80 citas, donde estudian el efecto de la diversificación de las empresas alemanas, japonesas y del Reino Unido. Por último el quinto artículo más citado es el de Klapper y Love (2004), que estudia el nivel de práctica del gobierno corporativo en compañías de mercados emergentes y demuestra que un buen gobierno corporativo está altamente correlacionado con una mejor actuación operativa y una mejor valoración del mercado. 


\subsection{AUTORES}

Para conocer el grado de especialización de los autores (Tabla 2), éstos se han clasificado según el número de artículos publicados siguiendo el criterio de gran autor (3-4 artículos), mediano ( 2 artículos) y pequeño (1 artículo). La élite son siete sobre un total de 642 autores que firman los 338 artículos de la muestra representando un $2,67 \%$ del total, mientras que los denominados autores medianos representan un $5,82 \%$ de la muestra. La gran mayoría de ellos, 588 , han publicado un solo artículo, lo que significa que un $91,50 \%$ de los autores que publican sobre GCI pueden ser clasificados de ocasionales.

Tabla 2: Productividad de los autores

\begin{tabular}{|l|c|c|c|}
\hline Tipología de autor & $\begin{array}{c}\mathbf{N}^{\mathbf{0}} \text { artículos } \\
\text { por autor }\end{array}$ & Autores & $\begin{array}{c}\text { \% sobre la } \\
\text { muestra }\end{array}$ \\
\hline \multirow{2yyn}{*}{ Gran autor } & 4 & 2 & \multirow{2}{*}{$2,67 \%$} \\
\cline { 2 - 3 } & 3 & 15 & $5,82 \%$ \\
\hline Mediano autor & 2 & 37 & $91,49 \%$ \\
\hline Autor ocasional & 1 & 588 & $100 \%$ \\
\hline \multicolumn{2}{|l}{ Número total de autores estudiados } & 642 & \\
\hline
\end{tabular}

Fuente: Elaboración propia a partir de los datos de ISI WoS.

Tal y como se puede ver en la tabla 2, los autores más productivos cuentan con cuatro artículos publicados cada uno de ellos. Estos autores han sido identificados como los profesores Trevor Buck e Igor Filatotchev quienes, además, destacan en su página web como su principal línea de investigación el GCI. Cabe señalar que, en este caso, los autores más productivos no coinciden con los autores de los trabajos más citados.

\subsection{COLABORACIÓN CIENTÍFICA}

Para poder estudiar la colaboración científica y su evolución se ha extraído el número de autores que firman cada uno de los artículos de cada uno de los años de la muestra (Tabla 3). Los resultados agrupados se muestran en la tabla 3, donde se puede apreciar cómo 236 artículos de los 338 publicados están firmados por más de un autor, por tanto se puede afirmar que existe colaboración científica, siendo los artículos con dos firmantes los más predominantes con 123 artículos. 
Tabla 3: Número de firmantes por artículo

\begin{tabular}{|l|c|}
\hline Número de firmantes & Frecuencia absoluta \\
\hline Trabajos con 1 firmante & 102 \\
\hline Trabajos con 2 firmante & 123 \\
\hline Trabajos con 3 firmante & 90 \\
\hline Trabajos con 4 firmante & 19 \\
\hline Trabajos con 5 firmante & 3 \\
\hline Trabajos con 6 firmante & 1 \\
\hline
\end{tabular}

Fuente: Elaboración propia a partir de los datos de ISI WoS.

De la relación entre el número de firmas totales y el número de artículos publicados resulta un promedio de 2,10 firmas por artículo.

El gráfico 2 muestra la evolución en el número de autores firmantes. Se puede apreciar cómo en las primeras publicaciones predomina la firma de un solo autor y que la colaboración entre dos autores solo comienzan a ser frecuente a partir de 1997.

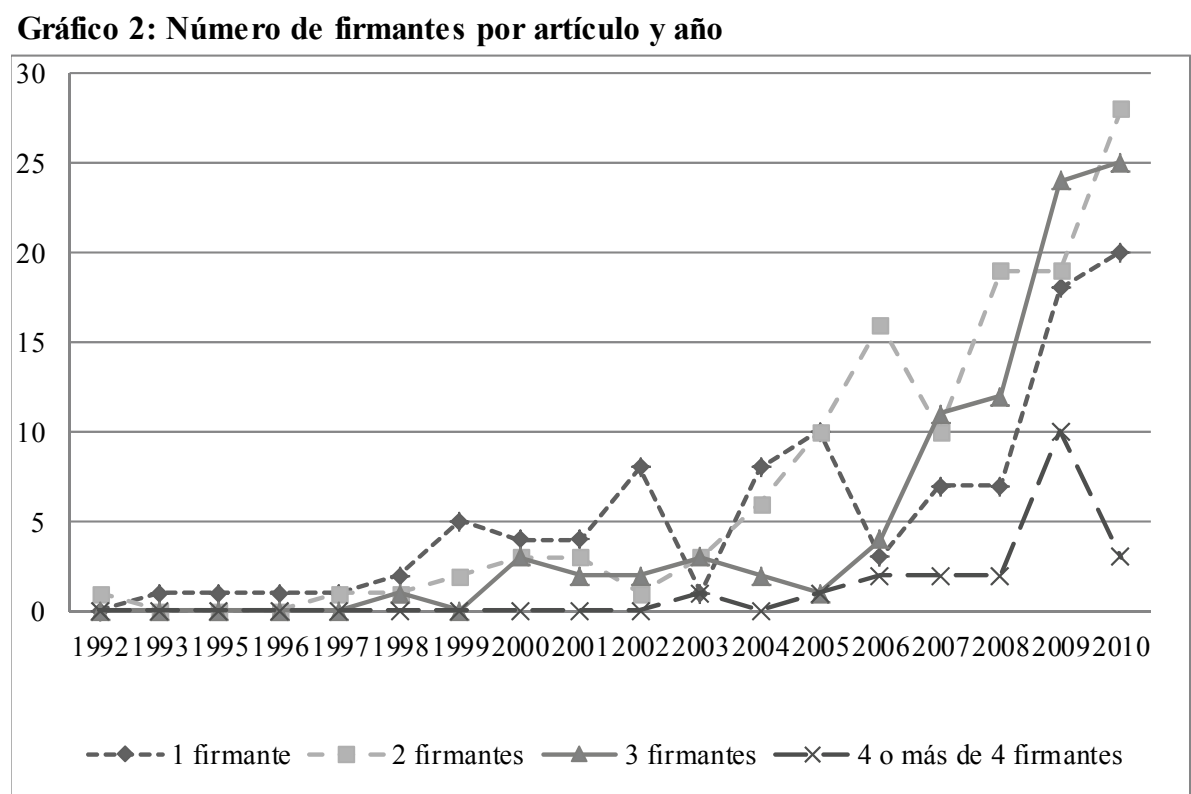

Fuente: Elaboración propia a partir de los datos de ISI WoS.

Los artículos firmados por tres autores no surgen hasta el año 1998, siendo frecuentes a partir del año 2000 y creciendo exponencialmente a partir del 2006. En cambio, los artículos firmados por cuatro o más autores, no se encuentran hasta el año 2003, y no se superan los cinco artículos publicados por años exceptuando el 2009 en el que se publicaron diez artículos con cuatro o más autores. Cabe 
destacar que los artículos publicados por un único autor predominan hasta el año 2005 , donde los artículos publicados por dos autores le superan en número de artículos publicados por año, y siendo superado también en el 2006 por los artículos publicados por tres autores.

\subsection{REVISTAS}

Los 338 artículos que conforman la muestra han sido publicados en 156 revistas científicas diferentes, siendo las que más artículos se han publicado sobre GCI: Journal of International Business Studies (26 artículos), Corporate Governance: An International Review (25 artículos) y Journal of Business Ethics (19 artículos). Las tres revistas representan el 20,77\% de la producción científica publicada (Tabla 4).

Tabla 4: Revistas más populares entre los autores para publicar

\begin{tabular}{|l|c|c|c|c|l|}
\hline Revistas & $\begin{array}{c}\text { Número } \\
\text { de } \\
\text { artículos } \\
\text { publicados }\end{array}$ & $\begin{array}{c}\text { Porcentaj } \\
\text { e de } \\
\text { artículos } \\
\text { publicado } \\
\text { s }\end{array}$ & $\begin{array}{c}\text { Porcentaje } \\
\text { acumulado }\end{array}$ & $\begin{array}{c}\text { Nivel de impacto } \\
\text { de re vis ta JCR } \\
\mathbf{2 0 0 9} \text { (Factor } \\
\text { impacto 5 años) }\end{array}$ & $\begin{array}{l}\text { Área de } \\
\text { conocimiento }\end{array}$ \\
\hline $\begin{array}{l}\text { Journal of International Business } \\
\text { Studies }\end{array}$ & 26 & $7,76 \%$ & $7,76 \%$ & 5,727 & Dirección de empresas. \\
\hline $\begin{array}{l}\text { Corporate Governance an International } \\
\text { Review }\end{array}$ & 25 & $7,39 \%$ & $15,15 \%$ & 3,117 & Dirección de empresas. \\
\hline Journal of Business Ethics & 19 & $5,62 \%$ & $20,77 \%$ & 1,692 & Ética. \\
\hline Journal of Banking \& Finance & 15 & $4,43 \%$ & $25,20 \%$ & 2,2 & Finanzas y contabilidad. \\
\hline Journal of Financial Economics & 13 & $3,84 \%$ & $29,04 \%$ & 5,675 & Finanzas y contabilidad. \\
\hline Journal of Finance & 7 & $2,07 \%$ & $31,11 \%$ & 6,536 & Finanzas y contabilidad. \\
\hline International Business Review & 6 & $1,77 \%$ & $32,88 \%$ & No disponible & Negocios. \\
\hline Journal of Corporate Finance & 6 & $1,77 \%$ & $34,65 \%$ & 2,073 & Dirección de empresas. \\
\hline Review of Financial Studies & 6 & $1,77 \%$ & $36,42 \%$ & 4,465 & Dirección de empresas. \\
\hline $\begin{array}{l}\text { Número total de artículos recogidos en esta } \\
\text { tabla }\end{array}$ & 123 & $36,42 \%$ & & & \\
\hline
\end{tabular}

Fuente: Elaboración propia a partir de los datos de ISI WoS.

El área de conocimiento de las revistas que mayoritariamente publican estos artículos está reflejado en la última columna de la Tabla 4 . Se observa que la mayoría de estas publicaciones se encuentran especializadas en la dirección de empresas y negocio. A continuación están las revistas especializadas en finanzas, con las tres revistas más importantes del área: Journal of Banking \& Finance, Journal of Financial Economics y Journal of Finance, que además cuentan con un importante nivel de impacto según Journal Citation Report (JCR).

Teniendo en cuenta el área de conocimiento de estas revistas, parece ser que la investigación sobre GCI se está desarrollando desde un punto de vista de la 
dirección de empresas primero y de las finanzas segundo, siendo la investigación desde otras áreas como la ética y la legal reducida.

Aplicando la ley de Bradford a la distribución de los artículos publicados por revista y tomando como núcleo las revistas Journal of International Business Studies y Corporate Governance: An International Review, se han establecido seis zonas de distribución de los artículos. La elección del núcleo se justifica por tres motivos; el primero de ellos viene dado por el hecho de que ambas destacan en el número de artículos publicados, por lo que difícilmente se podría consultar la producción científica consultando una de ellas y descartando la otra. El segundo motivo es su carácter internacional y por el grado de especializada de estas revistas en las comparativas internacionales. Por último, Corporate Governance: An International Review es una revista especialista en el tema. Una vez establecido el núcleo, éste ha determinado la magnitud de las zonas lo que ha establecido seis zonas de distribución de artículos (Tabla 5).

Tabla 5: Productividad de las revistas

\begin{tabular}{|c|c|c|c|c|c|c|}
\hline & $\begin{array}{c}\text { Tabla 8: } \\
\text { Número de } \\
\text { revistas }\end{array}$ & $\begin{array}{c}\text { Número de } \\
\text { artículos }\end{array}$ & $\begin{array}{c}\text { Porcentaje } \\
\text { de re vistas } \\
\text { por zona }\end{array}$ & $\begin{array}{c}\text { Porcentaje } \\
\text { acumulado } \\
\text { de revis tas }\end{array}$ & $\begin{array}{c}\text { Porcentaje } \\
\text { de } \\
\text { artículos } \\
\text { por zona }\end{array}$ & $\begin{array}{c}\text { Porce ntaje } \\
\text { acumulado } \\
\text { de } \\
\text { artículos }\end{array}$ \\
\hline Núcleo & 2 & 51 & $1,28 \%$ & $1,28 \%$ & $15,09 \%$ & $15,09 \%$ \\
\hline Zona 1 & 3 & 47 & $1,92 \%$ & $3,21 \%$ & $13,91 \%$ & $28,99 \%$ \\
\hline Zona 2 & 10 & 49 & $6,41 \%$ & $9,62 \%$ & $14,50 \%$ & $43,49 \%$ \\
\hline Zona 3 & 19 & 49 & $12,18 \%$ & $21,79 \%$ & $14,50 \%$ & $57,99 \%$ \\
\hline Zona 4 & 31 & 49 & $19,87 \%$ & $41,67 \%$ & $14,50 \%$ & $72,49 \%$ \\
\hline Zona 5 & 46 & 48 & $29,49 \%$ & $71,15 \%$ & $14,20 \%$ & $86,69 \%$ \\
\hline Zona 6 & 45 & 45 & $28,85 \%$ & $100,00 \%$ & $13,31 \%$ & $100,00 \%$ \\
\hline
\end{tabular}

Fuente: Elaboración propia a partir de los datos de ISI WoS.

Para ejemplificar la dispersión de las revistas se analiza el caso más extremo. Se tendrían que leer 45 revistas (las pertenecientes a la zona 6) para consultar los mismos artículos sobre Gobierno Corporativo Internacional que utilizando como fuente de información las dos revistas del núcleo.

\section{CONCLUSIONES}

Este trabajo ha realizado un estudio de la producción científica sobre la GCI de los últimos 20 años disponible en ISI WoS, aportando resultados que muestran su evolución en el tiempo. Una de las primeras conclusiones que se obtiene es que dicha producción sigue claramente la ley de crecimiento exponencial de Price, con un número 
exponencialmente creciente de artículos publicados año tras año. Estos resultados estarían en línea con los obtenidos por Richart et al. (2011) por lo que la producción científica sobre GCI, a pesar de ser una subdisciplina que nació varías décadas más tarde, sigue la misma tendencia que la producción en Gobierno Corporativo.

Tal y como señalaban Filatochev y Boyd (2009) esta disciplina no se generalizó hasta mediados de los noventa pero se ha podido comprobar cómo a partir de entonces se ha multiplicado el interés por su investigación alcanzando su punto álgido precisamente en el último año que se ha analizado. Cabe esperar que esta tendencia creciente continúe hasta llegar al punto de saturación de la disciplina hecho que con la información disponible no se puede determinar.

Del análisis de los artículos más citados se puede concluir que los artículos publicados con posterioridad al año 2004 son los más citados, incluso sobre artículos más antiguos. Este hecho mostraría que la investigación actual sobre GCI se está desarrollando a partir de los artículos publicados con posterioridad a dicha fecha.

El trabajo también señala a los dos autores de referencia sobre gobierno corporativo internacional, tanto por el número de artículos publicados como por el hecho de que su principal línea de investigación es precisamente la del tema estudiado. Estos autores, vale la pena destacar sus nombres una vez más, son los profesores Trevor Buck e Igor Filatotchev. A pesar de ser los más productivos no están entre los autores más citados, siendo éstos Ray Ball, S.P Kothari y Ashok Robin.

En cuanto al análisis de las revistas, se ha podido constatar que las más utilizadas para la publicación son aquellas que están circunscritas al ámbito de la dirección de empresas y las finanzas, con un carácter marcadamente internacional. Se puede concluir, por tanto, que la investigación se realiza mayoritariamente desde la aproximación de la dirección de empresas. También se ha constatado que el Journal of International Business Studies y Corporate Governance: An International Review son las principales revistas que recogen la producción científica, por lo que son las dos revistas principales de consulta para estar al día de los avances de la investigación.

\section{REFERENCIAS BIBLIOGRÁFICAS}

BALL, R., KOTHARI, S.P.; ROBIN, A. "The effect of international institutional factors on properties of accounting earnings". Journal of Accounting \& Economics, 2000, 29:1-51.

BECHT, M., BOLTON, P.; RÖEL, A. "Corporate Governance and Control". Handbook of the Economics of Finance, 2003, 1/1:1-109.

BEDCHUCK, L.; WEISBACH, M. "The State of Corporate Governance Research". Review of Financial Studies, 2010,23/3:939-961.

CHEW, D. Studies in International Corporate Finance and Governance Systems. Nueva York: Oxford. 
CORREA, M., GARCÍA, J.; ÚBEDA, R. "Análisis de la producción científica en Economía Financiera". Revista Española de Documentación Científica, 2009,32/2:83-104.

DEMB, A.; NEUBAUER, F. "The Corporate Board: Confronting the Paradoxes". Long Range Planning, 1992,25/3:9-20.

DENIS, D.; MCCONNELL, J. "International Corporate Governance". Journal of Financial and Quantitative Analysis, 2003, 38/1-36.

DOIDGE, C., KAROLYI, A. Y STULZ, R. "Why are foreign firms listed in the US worth .more?". Journal of Financial Economics, 2004,71/2:205-238.

DURISIN, B.; PUZONE, F. "Maturation of Corporate Governance Research, 1993-2007: An Assessment". Corporate Governance: An International Review, 2009,17/3:266-291.

DYCK, A.; ZINGALES, L. "Private benefits of control: An international comparison". The Journal of Finance, 2004,59/2:537-600.

FILATOTCHEV, I.; BOYD, B. "Taking Stock of Corporate Governance Research While Looking to the future". Corporate Governance: An International Review, 2009,17/3:261-262.

JENSEN, M.; MECKLING, W. "Theory of the firm: Managerial Behavior, Agency Cost and Ownership Structure". Journal of Financial Economics, 1976,3/4:305-360.

KLAPPER, L. LOVE, I. "Corporate Governance, Investor Protection and Performance in Emerging Markets". Journal of Finance, 2004,10/5:703-728.

LA PORTA, R.; LOPEZ DE SILANES, F.; SHLEIFER, A. "Law and Finance". Journal of Political Economy, 1985, 106:1113-1155.

LINS, K.; SERVAES, H. "International Evidence on the Value of Corporate Diversification". Journal of Finance, 1999,54/6:2215-2237.

MeYER, K., Estrin, S., BHAUMIK, K.; PENG, M. Institutions, resources, and entry strategies in emerging economies. Strategic Management Journal,2009,30/1:61-80.

RICHART, A.; MARTÍNEZ-BLASCO, M.; GARCÍA-BLANDÓN, J. “Análisis de la producción científica sobre Gobierno Corporativo a través de ISI Web of Science". Revista Española de Documentación Científica, 2011,34/80-101.

SHLEIFER, A.; VISHNY, R. (1997) A survey of Corporate Governance. The Journal of Finance, 1997,52/2:737-783. 